\title{
CRISPRED: A data pipeline for the CRISP imaging spectropolarimeter
}

\author{
J. de la Cruz Rodríguez ${ }^{1,2}$, M. G. Löfdahl ${ }^{1}$, P. Sütterlin ${ }^{1}$, T. Hillberg ${ }^{1}$, and L. Rouppe van der Voort ${ }^{3}$ \\ ${ }^{1}$ Institute for Solar Physics, Dept. of Astronomy, Stockholm University, Albanova University Center, 10691 Stockholm, Sweden \\ e-mail: jaime@astro.su.se \\ 2 Department of Physics and Astronomy, Uppsala University, Box 516, 75120 Uppsala, Sweden \\ ${ }^{3}$ Institute of Theoretical Astrophysics, University of Oslo, PO Box 1029 Blindern, 0315 Oslo, Norway
}

Received 1 June 2014 / Accepted 28 October 2014

\begin{abstract}
The production of science-ready data from major solar telescopes requires expertise beyond that of the typical observer. This is a consequence of the increasing complexity of instruments and observing sequences, which require calibrations and corrections for instrumental and seeing effects that are not only difficult to measure, but are also coupled in ways that require careful analysis in the design of the correction procedures. Modern space-based telescopes have data-processing pipelines capable of routinely producing well-characterized data products. High resolution imaging spectropolarimeters at ground-based telescopes need similar data pipelines. We present new methods for flat-fielding spectropolarimetric data acquired with telecentric Fabry-Perot instruments and a new approach for accurate camera co-alignment for image restoration. We document a procedure that forms the basis of current stateof-the-art processing of data from the CRISP imaging spectropolarimeter at the Swedish $1 \mathrm{~m}$ Solar Telescope (SST). By collecting, implementing, and testing a suite of computer programs, we have defined a data reduction pipeline for this instrument. This pipeline, CRISPRED, streamlines the process of making science-ready data. It is implemented and operated in IDL, with time-consuming steps delegated to C. CRISPRED will also be the basis for the data pipeline of the forthcoming CHROMIS instrument.
\end{abstract}

Key words. techniques: imaging spectroscopy - techniques: image processing - instrumentation: high angular resolution instrumentation: polarimeters

\section{Introduction}

During the past 20 years, a list of Fabry-Pérot interferometers (FPI) have been developed and installed in many groundbased facilities and airborne missions, e.g., TESOS at the VTT (Kentischer et al. 1998; Tritschler et al. 2002), IBIS at the Dunn Solar Telescope (Cavallini 2006), CRISP at the Swedish $1 \mathrm{~m}$ Solar Telescope (SST; Scharmer 2006), IMAX in the Sunrise mission (Martínez Pillet et al. 2011), GFPI at Gregor (Puschmann et al. 2013) and many more that are forthcoming. These instruments allow acquisition of spectropolarimetric data of a relatively large field of view (FOV) with very high cadence, very high spatial resolution, and an acceptable wavelength coverage (e.g., Tritschler et al. 2004; Cauzzi et al. 2008; Bello González \& Kneer 2008; Scharmer et al. 2008; Steiner et al. 2010; De Pontieu et al. 2012, among others).

In principle, FPIs can be mounted in telecentric (TESOS, CRISP) or collimated setup (IBIS, GFPI, IMAX). Most difficulties related to the processing of data from FPI instruments arise from microscopic corrugations in the surface of the etalons. In telecentric configuration, these aberrations are close to the focal plane, and therefore, the resulting data suffer from undesired wavelength shifts across the FOV. The unavoidable presence of atmospheric distortions from the ground further complicates the problem by introducing differential motions and aberrations in the image: between acquisitions, solar features are moved and distorted on the FOV, so there is not a unique wavelength shift (or pixel) that can be associated with that feature as it may have sampled a range of pixels in the different exposures and wavelengths. Image restoration techniques currently in widespread use for solar data are not designed to deal with such wavelength inhomogeneities, and often one has to resort to using approximations that make the images smoother prior to image restoration (e.g., Schnerr et al. 2011). In this paper, we propose a new method to accurately perform image restoration on datasets acquired with FPIs in telecentric setup.

Furthermore, a recurring problem inherent to most of the instruments mentioned above has been the lack of well established methods and techniques to properly reduce and process the resulting datasets, although some efforts have been made (e.g., Reardon \& Cavallini 2008; Schnerr et al. 2011), and certain corrections are standard to all instruments: dark current and flat-field correction, pre-filter compensation, and (in most cases) image restoration.

The task of going from raw data to science-ready data is beyond the skills of the majority of potential users because of the complexity of the data calibration and required compensation for seeing degradation. There is thus a strong incentive to relieve solar scientists from the burden of gaining expert knowledge about state-of-the-art instrumentation by supplying them with easy to use data pipelines with well-characterized data products. Welldesigned data pipelines allow such instrumentation to reach their full scientific potential, while minimizing the efforts of the user and opening up access to the facility to inexperienced users. Such pipelines will also be necessary in the future when solar telescopes are routinely operated in service mode and are required to deliver science-ready data to scientists (Reardon et al. 2009). The European solar community has recognized the need for such pipelines and therefore have included pipeline development in the recently started EU-funded SOLARNET project. 
In this paper, we also describe CRISPRED ${ }^{1}$, a data reduction pipeline for CRISP. The pipeline started as a collection of scripts written by different (present as well as former) researchers at the Institute for Solar Physics (ISP), Uppsala University, and University of Oslo, coded in a variety of languages. These heterogeneous pieces of code have since been incorporated into an object-oriented IDL structure with computationally demanding parts performed in $\mathrm{C}$ subprograms called as dynamically loadable modules (DLMs). Early versions of CRISPRED have been used by several authors to reduce and prepare their data, given that a basic framework has been functional since 2011 (e.g., Watanabe et al. 2012; Scharmer et al. 2013; de la Cruz Rodríguez et al. 2013; Vissers et al. 2013; Sekse et al. 2013; Wedemeyer et al. 2013; Rouppe van der Voort \& de la Cruz Rodríguez 2013; Leenaarts et al. 2014).

A pipeline for an instrument like CRISP must be based on a detailed knowledge about the formation of the image data, including the various sources of measurement error and the calibrations that allow them to be characterized. Section 2 describes the image formation relevant for FPI-based imaging spectropolarimeters like CRISP.

The calibration and removal of the errors and contaminations are important parts of the pipeline. Newly developed methods are introduced in Sect. 3, before we describe the CRISPRED pipeline in Sect. 4. We end with a discussion on future developments in Sect. 5.

\section{Image formation and optical setup}

The signal that we want to detect with an imaging spectropolarimeter, intensity and polarization of visible light, is formed in the Sun's photosphere and chromosphere. The intensity pattern over the FOV is modulated by polarization optics and the final image is the convolution of this intensity pattern with the telescope PSF at the wavelength selected by narrowband (NB) filters.

The image formation is quite complex. Turbulence in the Earth's atmosphere degrades the image quality and causes spectral cross-talk. The telescope and the optical setup introduce undesirable polarization. The filter profiles vary over the FOV and introduce other unwanted effects. The following sections describe the image formation, including an overview of the most important deviations from the ideal case.

The optical setup and the details of the instrument are obviously important for the formation of the images. The CRISPRED pipeline is developed for SST/CRISP, so we provide a description of that particular instrument for the following discussion. We believe most of the CRISPRED methods described in later sections are easily adaptable to similar instruments but we will point out when a certain feature in the setup is necessary. More details of the CRISP setup can be found in the Appendix.

\subsection{Atmosphere and telescope}

Turbulence randomly mixes air with varying temperature (and therefore refractive index), causing perturbations in the wavefront phases that vary spatially with a timescale of ms. We refer the interested reader to Fried (1965) and Roddier (1999) for mathematical descriptions of these effects. Turbulence near the telescope aperture causes global motion and blurring of the image. Similar effects at higher altitudes $(\sim 10 \mathrm{~km})$ take the form of variations over the FOV, where the seeing causes rubber-sheet

\footnotetext{
1 http://dubshen . astro.su . se/wiki/index .php?title= CRISPRED
}

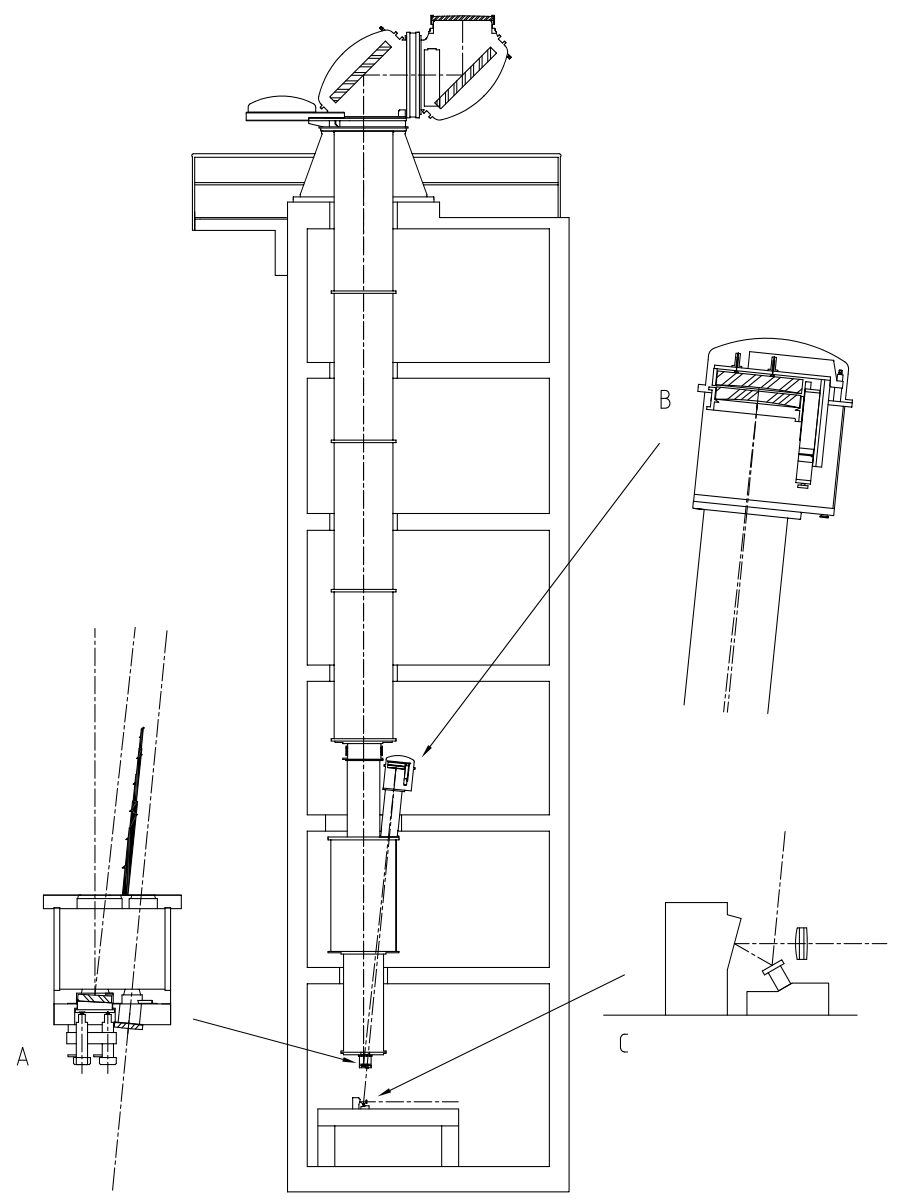

Fig. 1. Optical path of SST, from the $1 \mathrm{~m}$ lens, via the two alt-az mirrors, the field mirror on the bottom plate (inset A), the Schupmann corrector (inset B), the field lens and exit window (inset A), to the tip-tilt mirror, deformable mirror, and re-imaging lens on the optical table (inset C). The optical path continues in Fig. 2. Courtesy of Göran Scharmer.

deformations of the image and blurring that varies over small scales. Scattering from aerosols can produce a diffuse component that lowers the contrast. Particularly in calima conditions, when the Saharan air layer, an elevated (1000-5500 m) layer of dry air and mineral dust originating from the Saharan desert, forms over large parts of the northern equatorial Atlantic (e.g., Dunion \& Velden 2004). The polarizing effects from the atmosphere are visible in the blue sky but are small in direct sunlight.

The SST is located on the island of La Palma, a little less than $2400 \mathrm{~m}$ above sea level in a $17 \mathrm{~m}$ high tower. Figure 1 shows the optics of the telescope. Via the two $45^{\circ}$ turret alt-az mirrors, the beam is directed down through the vertical telescope tube to a primary focus on the bottom plate. The alt-az configuration causes image rotation and the rotation of the lens and two mirrors cause time-varying polarization (Selbing 2005), mostly far from focus. The lens itself is a singlet and therefore has enormous chromatic aberrations. These are removed by a Schupmann corrector, that is fed from a field mirror on the bottom plate of the tube and forms a new focus just outside an exit vacuum window behind a large baffle.

\subsection{Pre-CRISP optics}

We refer now to Fig. 2, where we show a schematic view of the optical setup below the telescope, the details of which are described in this section. 


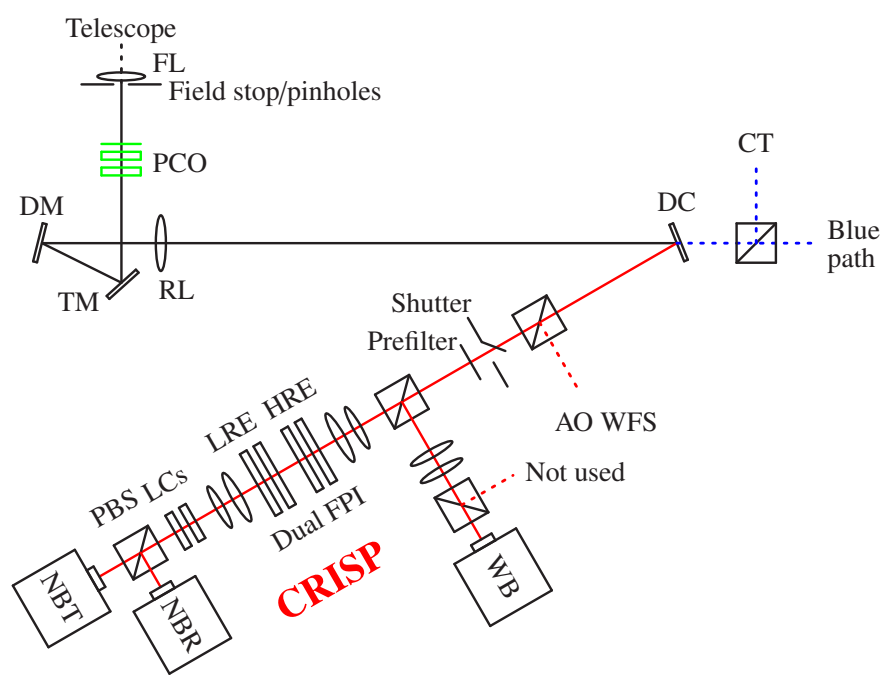

Fig. 2. Optics, from bottom of telescope (inset A in Fig. 1) to the CRISP. $\mathrm{FL}=$ field lens, $\mathrm{PCO}=$ polarization calibration optics (not in the beam during normal observations), $\mathrm{TM}=$ tip-tilt mirror, $\mathrm{DM}=$ deformable mirror, $\mathrm{RL}=$ reimaging lens, $\mathrm{DC}=$ dichroic beamsplitter, $\mathrm{CT}=$ correlation tracker, AO WFS $=$ adaptive optics wavefront sensor, FPI $=$ Fabry-Pérot interferometer, HRE $=$ high resolution etalons, LRE $=$ low resolution etalons, $\mathrm{WB}=$ wideband, $\mathrm{NBT}=$ narrowband transmitted, $\mathrm{NBR}=$ narrowband reflected, $\mathrm{LCs}=$ liquid crystal modulators, $\mathrm{PBS}=$ polarizing beam splitter.

The field lens (FL) is near the focus from the Schupmann corrector and it is designed to form a $34 \mathrm{~mm}$ diameter pupil image at the Adaptive Optics (AO) deformable mirror (DM). Near the Schupmann focus, various artificial targets and field stops can be slid into the beam. Just outside the science FOV, a part of the beam is reflected toward a wide-field wavefront sensor (WFS - not shown in Fig. 2), that can be used for monitoring the seeing profile above the telescope (Scharmer \& van Werkhoven 2010).

The AO has two parts. The correlation tracker (CT) measures image motion and uses the tip-tilt mirror (TM) to keep the image steady. The recently installed 85 microlens ShackHartmann WFS measures the phase of the wavefront at the pupil and tries to keep it flat by controlling the shape of the 85 electrode monomorph DM from CILAS at a rate of $2 \mathrm{kHz}$. This system (Scharmer et al., in prep.) is similar to the previous system by Scharmer et al. (2003), although it is an upgrade in both hardware and software.

Between the target slider and the TM, polarization calibration optics (PCO) can be inserted in the beam. There are three parts: a red filter, a rotatable linear polarizer, and a retarder. They are not in the beam during normal science observations.

The TM and DM together make the beam horizontal and send it through the reimaging lens (RL) toward the dichroic beamsplitter (DC), which splits the beam (at $500 \mathrm{~nm}$ ) into a red beam and a blue beam. The CRISP is in the red beam, behind a beamsplitter that sends a small fraction of the light to the AO WFS.

\subsection{CRISP}

The tunable filter of the CRisp Imaging Spectropolarimeter (CRISP, Scharmer 2006; Scharmer et al. 2008) is a dual FabryPérot interferometer consisting of two etalons mounted in tandem in a telecentric configuration. An etalon consists of two parallel reflecting plates, forming a cavity where the light undergoes multiple reflections. An infinite train of transmission peaks

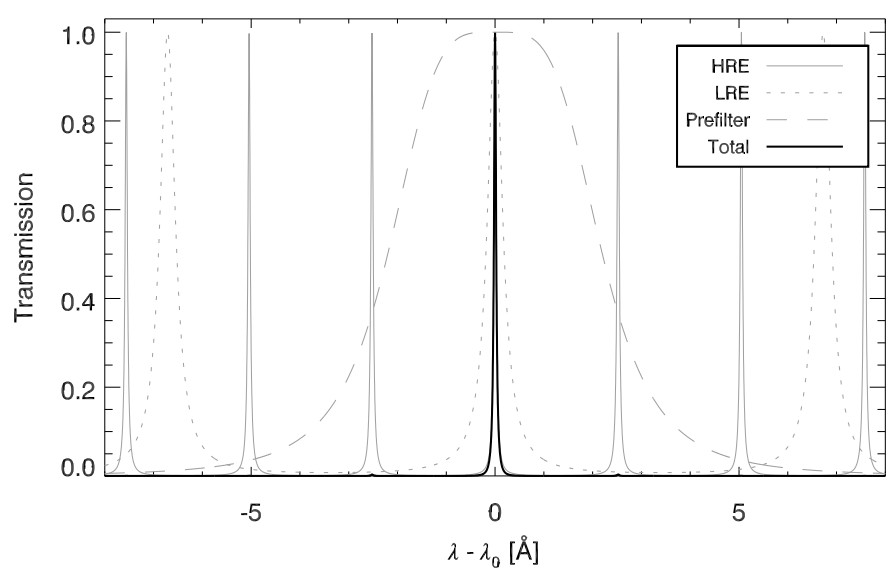

Fig. 3. Approximate CRISP transmission profile at $630 \mathrm{~nm}$, computed theoretically assuming perpendicular incidence of the beam with the surface of both etalons. The individual contributions from the high resolution etalon, the low resolution etalon, and the prefilter are shown along with the total transmission. All curves are normalized to unity.

is produced by multiple interference of light inside the cavity. The FPI produces quasi-monochromatic images by combining a high spectral resolution etalon (HRE) that defines the observed wavelength and a lower spectral resolution etalon (LRE) that suppresses the first few orders of secondary HRE transmission peaks. A prefilter limits the spectral range of the light that passes through the optical system, attenuating all higher order transmission peaks of both etalons (see Kentischer et al. 1998; Cavallini 2006; Scharmer 2006, and references therein).

Figure 3 shows the individual transmission curves for the two etalons, the prefilter and the total CRISP transmission. We note that the total transmission curve corresponds to that at the center of the prefilter. Observations in the outer flanks of the prefilter curve have larger contributions from the first few secondary transmission peaks.

The transmission profile of each etalon can be approximately computed for each ray as described by Kentischer et al. (1998),

$$
T(\delta \lambda, \delta R)=\frac{1}{1+\frac{4(R+\delta R)}{(1-R-\delta R)^{2}} \sin ^{2}\left(\frac{\Psi(\delta \lambda)}{2}\right)},
$$

where $R$ is the reflectivity of the etalon, and $\Psi$ is the phase difference of succeeding reflections between the two reflecting plates

$\Psi(\delta \lambda)=2 \cdot\left(\frac{2 \pi}{\lambda+\delta \lambda}\right) \cdot n \cdot D \cdot \cos \theta$

where $\lambda$ is the wavelength, $\delta \lambda$ is the cavity error ${ }^{2}, n$ is the refractive index of the cavity, $D$ is the nominal separation between the reflecting surfaces of the etalons, and $\theta$ is the angle of incidence of the beam.

The telecentric mount was chosen because it optimizes the image quality in the focal plane (see Scharmer 2006). However, this design introduces field-dependent variations in the transmission profile:

- Imperfections on the surface of the etalons influence the phase difference among successive reflections between the

2 The cavity error is really an error in the cavity separation $D$. Attributing it to the wavelength is mathematically equivalent $(\delta \lambda=$ $\delta D \cdot \lambda / D)$ and makes it immediately useful for correction of the data. 

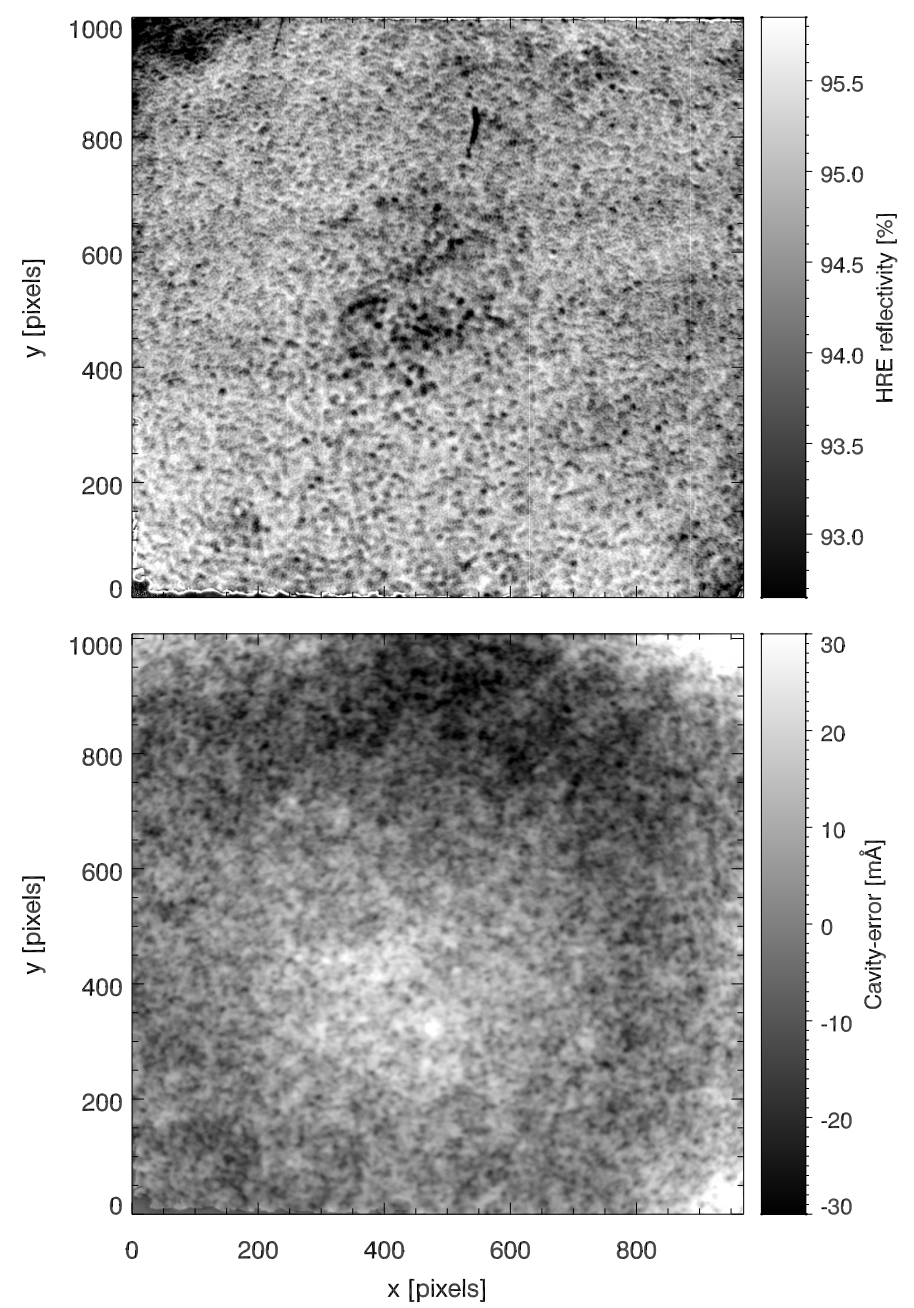

Fig. 4. Characterization of the CRISP instrument at $617 \mathrm{~nm}$. Top: reflectivity fluctuations of the high resolution etalon. Bottom: cavity-error map of the high resolution etalon.

reflecting plates of the etalons. Thus, the position of the central wavelength of the transmission profile varies over the etalon surfaces, producing field-dependent wavelength shifts of the observed spectral lines. These are commonly known as cavity errors (bottom panel in Fig. 4).

- Reflectivity variations in the coating of the etalons make the width of the transmission profile to change along the FOV. Commonly known as reflectivity errors (top panel in Fig. 4).

We discuss in detail the characterization of CRISP parameters and the treatment of cavity errors in Sects. 4.3 and 3.1.

The CRISP transmission profile depends critically on the reflectivity of the coating of the etalon: narrower profiles correspond to higher values of the reflectivity. Figure A.1 shows the full width half maximum (FWHM) of the CRISP transmission profile as a function of wavelength. To derive the FWHM, we have used the nominal reflectivity values of the etalons as measured by the manufacturer and Eq. (1).

Reimaging optics are placed before and after the etalons to make the beam slower at the location of the etalons, and to place the focus between the two etalons. After the second re-imaging, a pair of modulators ${ }^{3}$ produce four polarization states that are

\footnotetext{
3 The present modulators are a pair of nematic liquid crystals that change their states too slowly to finish during the covered phase of the
}

linear combinations of the four Stokes parameters. Finally, a linearly polarizing beam splitter separates the light into horizontal and vertical polarized components and sends them to the respective NB camera.

The CRISP instrument includes three Sarnoff CAM1M100 cameras with back-illuminated, thinned CCD detectors consisting of 1024 by $102416 \times 16 \mu \mathrm{m}^{2}$ pixels. One camera acquires wideband (WB) images directly from the prefilter and two cameras acquire NB images through the FPIs. After the prefilter wheel, a beam splitter sends $\sim 8 \%$ of the light to the WB channel and $\sim 92 \%$ to the NB channel. Placing the WB camera after the CRISP prefilter is crucial for the MOMFBD image restoration that is described in Sect. 4.4, because it relies on always having simultaneous WB images with a wavelength near the NB wavelength.

The three cameras are synchronized by use of an external shutter (chopper), to facilitate image restoration. The chopper revolves at $\sim 37 \mathrm{~Hz}$ with an exposure time of $\sim 17 \mathrm{~ms}$, using the covered $\sim 10 \mathrm{~ms}$ for read out.

\section{Newly developed methods}

In this section we describe methods of general use, that are original to this publication or have not appeared in the peer-reviewed literature.

\subsection{Flat-fielding and image restoration of wavelength inhomogeneous data}

In theory, corrections and calibrations to data should be applied following the optical path in reversed order (starting at the detector). Accordingly, image restoration (see Sect. 4.4 below) should be the very last correction to be applied to the data, after dark current, flat-field, polarimetric calibration, prefilter transmission, and wavelength shifts. The main problem is that we need to perform image restoration before the polarimetric calibration to ensure that all narrow-band states are perfectly co-aligned and de-stretched. We also need to perform image restoration before compensating for wavelength shifts (from the FPI) because otherwise atmospheric distortion would make the spectra spatially incoherent across the different line positions, as solar features are moved around by the seeing. The data processing described in this paper becomes highly complicated because we need to perform image restoration at earlier stages than we should, and cavity errors cannot be corrected prior to image-restoration.

Conceptually, it should be possible to perform a forward model fitting of the spectral line in each position within the FOV, including polarimetry as well as wavefront aberrations and geometrical distortions from anisoplanatism, and try to reproduce the observed data. In practice, this kind of modeling is very difficult to implement and the computations are likely to be demanding. Perhaps the techniques described by van Noort (2012) set the ground for additional developments in this direction.

Current implementations of image restoration deal with quasi-monochromatic data, where the distortions of the object (the Sun) are assumed to be due only to wavefront aberrations. The combined effect of cavity errors and a line profile imprints the images with a pattern of intensity fluctuations, resulting from

chopper. They are therefore operated with overdrive, making them very sensitive to temperature variations within the observing room. New, faster, polychromatic modulators based on ferroelectric liquid crystals (de Wijn et al. 2011) will be installed during the 2014 season. 


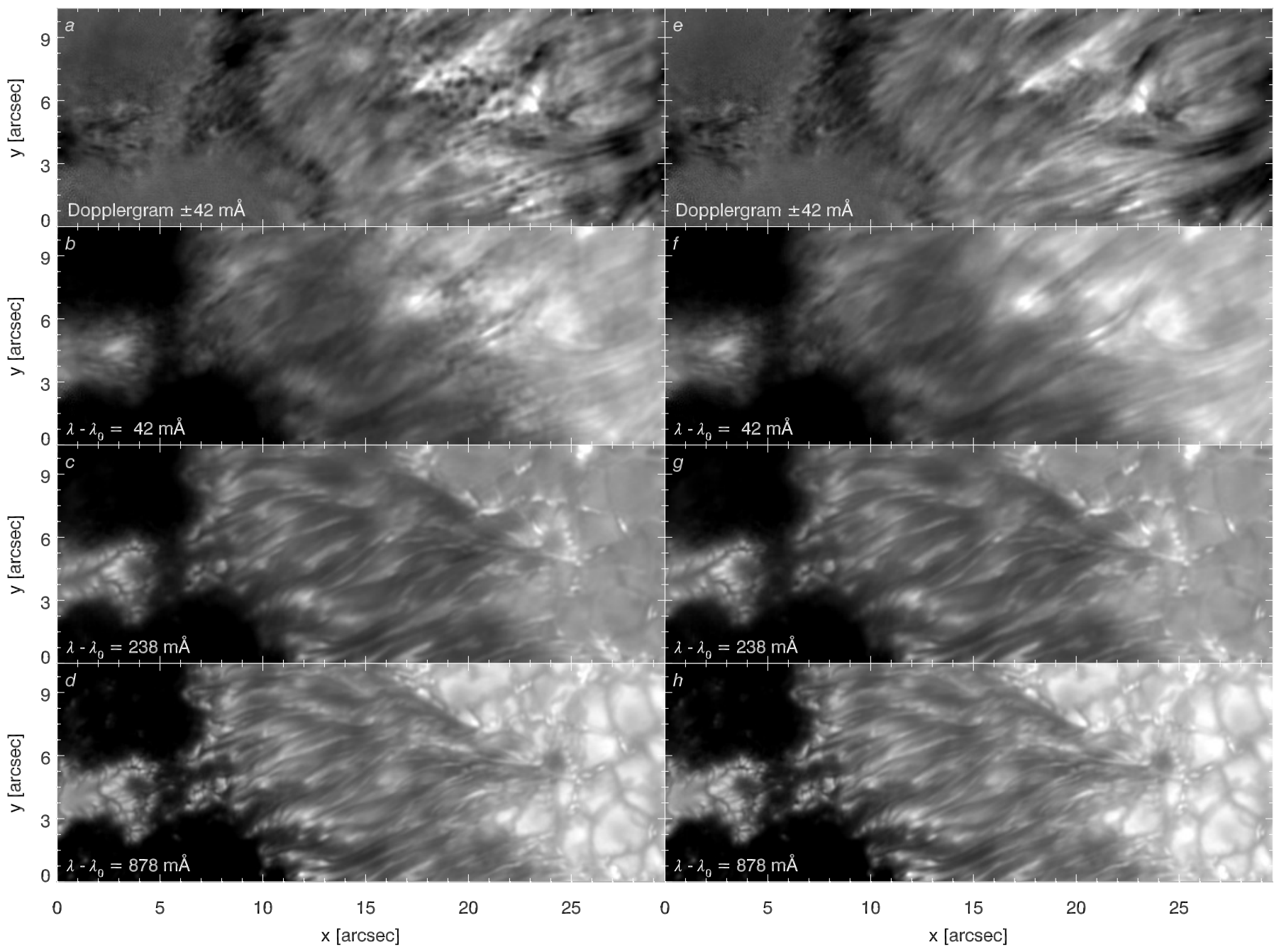

Fig. 5. Observations in the Mg I $\lambda 5173$ line. The top row correponds to Dopplergrams (calculated from images at $\pm 42 \mathrm{~m} \AA$ ). The other panels, in descending order, show red wing images, $\lambda-\lambda_{0}=42,238$, and $878 \mathrm{~m} \AA$, respectively. The images in the left column were restored with the flat-fielding scheme by Schnerr et al. (2011). Artifacts due to cavity errors appear as small-scale grains, clearly visible in panels a) and b). In contrast, no such errors are visible in the right column, corresponding to images restored with our new method.

the wavelength shift of the transmission profile, violating this assumption. The effect is strong enough to disturb the wavefront sensing process, particularly in bad seeing. Deconvolution of the cavity-error pattern causes further artifacts in the restored images.

Without any kind of wavelength shift compensation, the restored images show a background of strong artifacts that are somewhat correlated with the distribution of wavelength shifts. This pattern is particularly visible when the gradient of the spectral profile is steep, and it disappears in the continuum. These are the issues that make the data reduction process entangled and complicated for instruments like CRISP and TESOS.

Schnerr et al. (2011) proposed an approximate solution: once the cavity-error map is known, one can use the quiet-Sun average profile, present in the flat-field data, to approximately correct the effects of cavity errors, assuming that the slope of the resolved pixel-to-pixel profile is somewhat similar to that from the quietSun average. This approach allowed them to easily compute a correction that makes the image smoother for the image restoration process. Afterward, the restored images would be multiplied by the ratio between this incorrect flat-field dataset and the one corrected for cavity errors (where the quiet-Sun profile is removed).
Obviously, there are limitations to this approach. A spectrum from a sunspot is hardly similar to a quiet-Sun mean profile, in strength, width and shape, but this approximation seems to work well when the seeing conditions are excellent, probably because the contrast of the images dominates over the intensity fluctuations introduced by cavity errors. Normally, the effect of cavity errors is stronger at shorter wavelengths, because the ratio $D / \lambda$ in Eq. (2) is larger.

In Fig. 5 we illustrate the effect with a CRISP dataset acquired in the Mg I 5173 line, where the flat-fielding scheme proposed by Schnerr et al. (2011) leads to small-scale artifacts in some restored images (left column), especially close to line center where the line profile is very steep (panel $b$ and the Dopplergram in panel $a$ ). We note that artifacts will preferentially appear in the presence of a steep line profile and low image contrast and therefore not necessarily in all line positions (e.g., panels $c$ and $d$ ) or even within the entire line scan.

In this study, we present a more accurate way to deal with the flat-fielding of non-monochromatic images for image restoration. The idea is to use non-restored data to measure the observed spectra across the FOV, and then calculate the corrections needed to compensate for intensity fluctuations introduced by the cavity error. For each line scan, we add all the images acquired in 
a burst for each wavelength, resulting in a data-cube with line profiles for each pixel, $I(\lambda, x, y)$.

These profiles can be shifted by interpolation, producing the data-cube $I^{*}(\lambda-\delta \lambda(x, y), x, y)$, where $\delta \lambda(x, y)$ is a cavity map, estimated as in Sect. 4.3 below (see also Fig. 4 ). The corrected flatfield, that will be used to perform the image processing, $F_{\text {corr }}$, can then be constructed using the cavity-error-free flat-field images, $F_{\text {cef }}$, multiplied by the ratio between the shifted and unshifted profiles,

$$
F_{\text {corr }}(\lambda, x, y)=F_{\text {cef }}(\lambda, x, y) \frac{I(\lambda, x, y)}{I^{*}(\lambda-\delta \lambda(x, y), x, y)} .
$$

This method should provide cavity-error-free images because it effectively shifts the observed line profile so that the wavelength is constant within a frame. In our tests, however, we noticed that some residual distortion could be introduced in the restored images because the spectra from our summed (unprocessed) data are not free of atmospheric distortions.

To overcome this inconvenience, a mathematical trick can be used (Scharmer 2014, priv. comm.). Because of the limited spatial extent (a few arcsec) of the PSF, the image restoration is only affected by the presence of fluctuations at high spatial frequencies, whereas it is rather insensitive to the presence of large scale variations. This strongly limits the distance over which intensity information from different wavelengths are mixed. Thus, it is the small-scale fluctuations that cause artifacts in the deconvolution because they are discontinuous to a certain extent, whereas the large-scale fluctuations do not introduce this problem.

By correcting only the high frequency fluctuations, the profile is shifted by a significantly smaller amount and potential residual distortions remain invisible. The low frequency component can be corrected, once the images have been restored from wavefront aberrations. In addition, the correction proposed in Eq. (3) is strictly more appropriate since we are assuming that the slope of the line profile at any given wavelength has linear dependence. This assumption becomes less accurate when corrections are large.

We illustrate the concept in Fig. 6, which shows the cavityerror map from Fig. 4, decomposed into two components: the low spatial frequencies (9.16 $\mathrm{m} \AA$ amplitude rms contrast) and the high spatial frequencies (5.07 $\mathrm{m} \AA$ amplitide rms contrast). Both panels are scaled between $\Delta \lambda= \pm 30 \mathrm{~m} \AA$. We note the much smaller amplitude of the high frequency component. We show the results of a restoration performed with our new method in the right column of Fig. 5. The Dopplergram and all images are free from visible cavity-error artifacts.

\subsection{Pinhole calibration}

As reference for inter-camera alignment, we use a pinhole array that is mounted at the Schupmann focus. Most telescopes should have an early focus where such a target can be mounted. Multiple images of this array are routinely collected every observing day in the same way as flat-field data, and co-added separately for each camera and state. A grid of about $12 \times 12$ pinholes fit in the detector FOV, see Fig. 7. Removing the pinholes closest to the edges leaves about $10 \times 10$ pinholes for further processing.

The maximum intensities of the pinholes are first used for finding out the relative orientation (rotation and mirroring) of the cameras, as well as alignment errors on the order of the grid spacing or larger (which should never happen). This works because the amount of light passing through the different holes

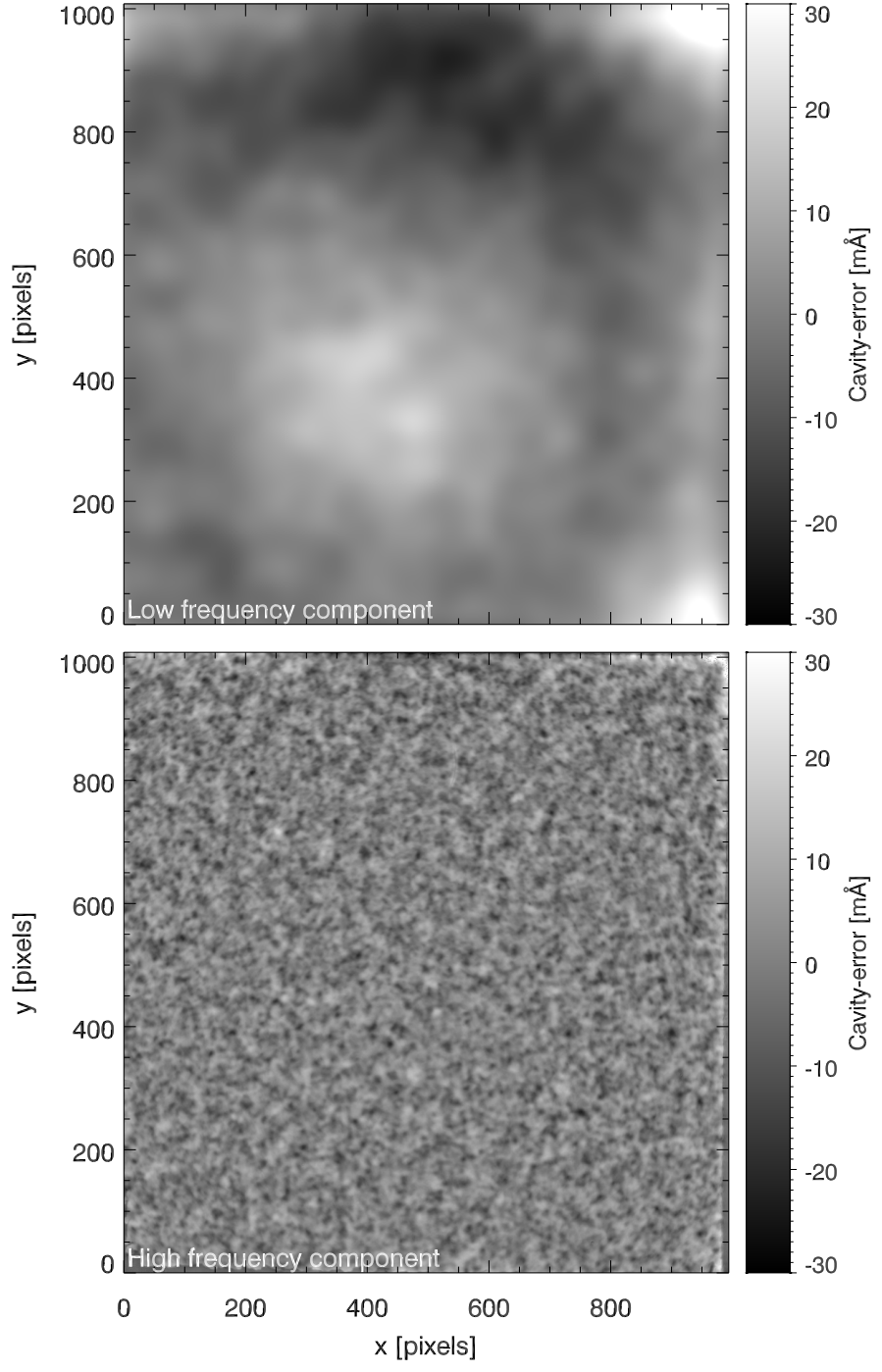

Fig. 6. The cavity-error map from Fig. 4, decomposed into low spatial frequencies (top) and high spatial frequencies (bottom).

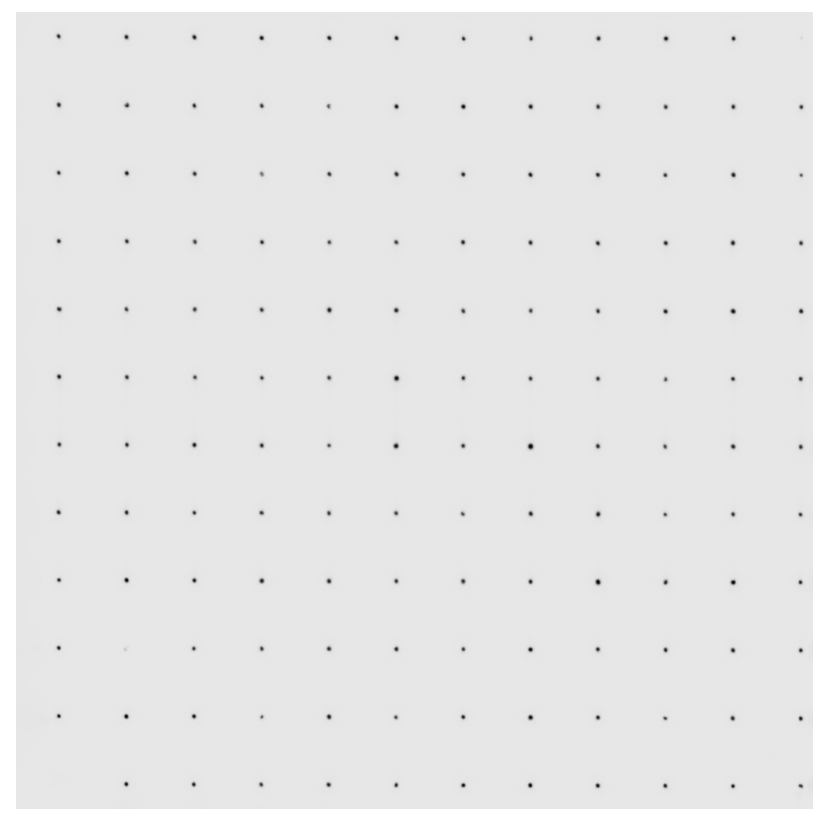

Fig. 7. A $1024 \times 1024$ pixel $\left(600^{\prime} 4 \times 60{ }^{\prime} 4\right)$ image of the pinhole array, inverse intensity scale. 
depends on size and blockage by dust or dirt, forming a distinct pattern.

The summed pinhole images have negligible solar structure within the small area of the holes, and therefore the object can be assumed to be the same in all cameras and states. By individually MOMFBD-processing subfields centered on each of the pinholes, we estimate field-dependent alignment parameters that represent the alignment as a function of position in the FOV. Two-dimensional fits to these estimated values are stored to disk, where the MOMFBD program can look up the shifts needed to align the cameras at a particular location.

Over the last ten years, the non-MOMFBD parts of this procedure have been implemented in ANA, shell script, python, and $\mathrm{C}++$. When we re-wrote it in IDL, we also upgraded the procedure slightly.

Some pinholes are completely or partially blocked, giving bad MOMFBD inversions, see gaps in the pinhole grid in Fig. 7. Therefore, we only process pinholes brighter than a threshold, reducing the number of outliers included in the fit.

Two problems with the pinhole processing were solved by methods resembling what was done by Löfdahl \& Scharmer (2012), who were able to measure not only focus terms but also higher order aberrations from pinhole data. We now sum the pinholes with sub-pixel alignment after filling in bad pixels with interpolation. This solves the problem with sub-pixel jitter between exposures, which can violate the assumption of a common object if the sums do not involve the same exposures for all cameras. We then refine the dark level of each subfield individually. When all the energy in an image is concentrated to a few pixels, also small errors in the dark level can make problems for the assumption of a common object. Using the WB as the anchor channel, in the old procedure, pinhole MOMFBD was run separately for $\mathrm{WB}+\mathrm{NBT}$ and $\mathrm{WB}+\mathrm{NBR}$, respectively. Now we run $\mathrm{WB}+\mathrm{NBT}+\mathrm{NBT}$, resulting in (at least potentially) more constrained inversions.

These changes improved the consistency of the estimated offset surfaces between different states. This strengthened the case for another change in the procedure. Earlier, we have mostly measured the NB pinhole shifts with respect to the WB, not only for each NB camera, but also for each combination of camera, prefilter, wavelength tuning, and modulator state. This requires very large amounts of pinhole data to be collected for observing sequences with many wavelength points. The pipeline will still perform the pinhole calibration for all data that are collected but the recommendation is now to collect data only for a single state in each scan (prefilter), and then use the measured offsets for all states. An exception will be made for the very broadest lines, where data should be collected for a few wavelength tunings and the offsets then interpolated.

\subsection{Image scale}

Changes on the optical table can change the image scale significantly. We also discovered recently that the CRISP prefilters have a slight optical power, enough to move the focus by a few $\mathrm{mm}$ (optionally compensated for when observing with an extra focus term on the DM) and also change the image scale slightly. The pinhole array mounted at the Shupmann focus, used as a reference for camera alignment (see Fig. 7), is therefore used as the reference also for determining the image scale in angular units per pixel.

To serve as such a reference, the pinhole grid spacing must be known in angular units. By use of a code developed by Norén (2013) we measured the image scale in the CRISP WB camera

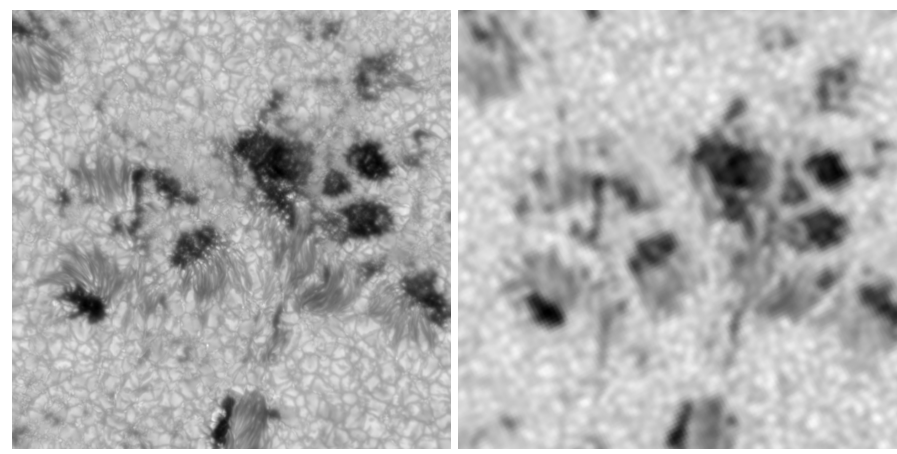

Fig. 8. Co-temporal and co-spatial $558 \mathrm{~nm}$ SST/CRISP (left) and $617 \mathrm{~nm}$ SDO/HMI (right) images used for image scale measurements. The FOV covers part of AR1589, which was near disk center on 2012$10-15$.

by comparison with approximately co-temporal $617 \mathrm{~nm}$ continuum images from SDO/HMI (Schou et al. 2012). Images of AR1589, an active region with many high-contrast features distributed over the SST FOV, were collected on 2012-10-15 and MFBD-restored in groups of $\sim 100$ exposure, then downsampled to the same resolution as the HMI images (sample images are shown in Fig. 8). We measured in two wavelengths, through the 558 and $630 \mathrm{~nm}$ CRISP prefilters, and got image scales 0.'05900/pixel and 0.'05876/pixel, respectively. The accuracy of these measurements is determined by two factors. The HMI image scale is known to a relative error of $0.07 \%$ or better, mostly limited by the value adopted for the solar radius (Schou 2013, priv. comm.). The standard deviations of the image scale measurements performed separately on about 150 SST images is on the same order of magnitude, resulting in a combined uncertainty of about $0.1 \%$.

As part of the CRISPRED pipeline, we measure the pinhole spacing (in pixels) by measuring the individual positions of each pinhole in the FOV and fitting (using MPFIT) those positions to a model with five parameters: the coordinates of the pinhole nearest the origin, the grid spacing in the two grid axis directions, and a rotation angle. Assuming we can measure the pinhole positions to better than pixel accuracy, over the $\sim 1000$ pixel detector size we should get accuracy that is significantly better than the $0.1 \%$ of the SST/HMI image scale calibration.

Measuring the pinhole grid spacing in pixels in pinhole array images, collected with the same cameras and on the same day as the active region data above, gives 86.75 pixels and 87.04 pixels, respectively, in the two wavelengths. This means that the measurements agree on a grid spacing of 5'. $12\left(5^{\prime \prime} .116 \pm 0\right.$. .'005) for both wavelengths. This number is used in the pipeline as the reference for calculating the image scale in all cameras. The so calibrated image scale is then used by the MOMFBD program (Sect. 4.4.1) and should also be used for interpretation of the data.

\section{The CRISPRED pipeline}

In this section we describe the corrections involved in the data reduction of CRISP, but most of them are common to instruments with similar characteristics.

Figure 9 illustrates the data flow of the CRISPRED pipeline. All the details are provided in the following sections, but we include here a very brief summary of the data flow. 
A\&A 573, A40 (2015)

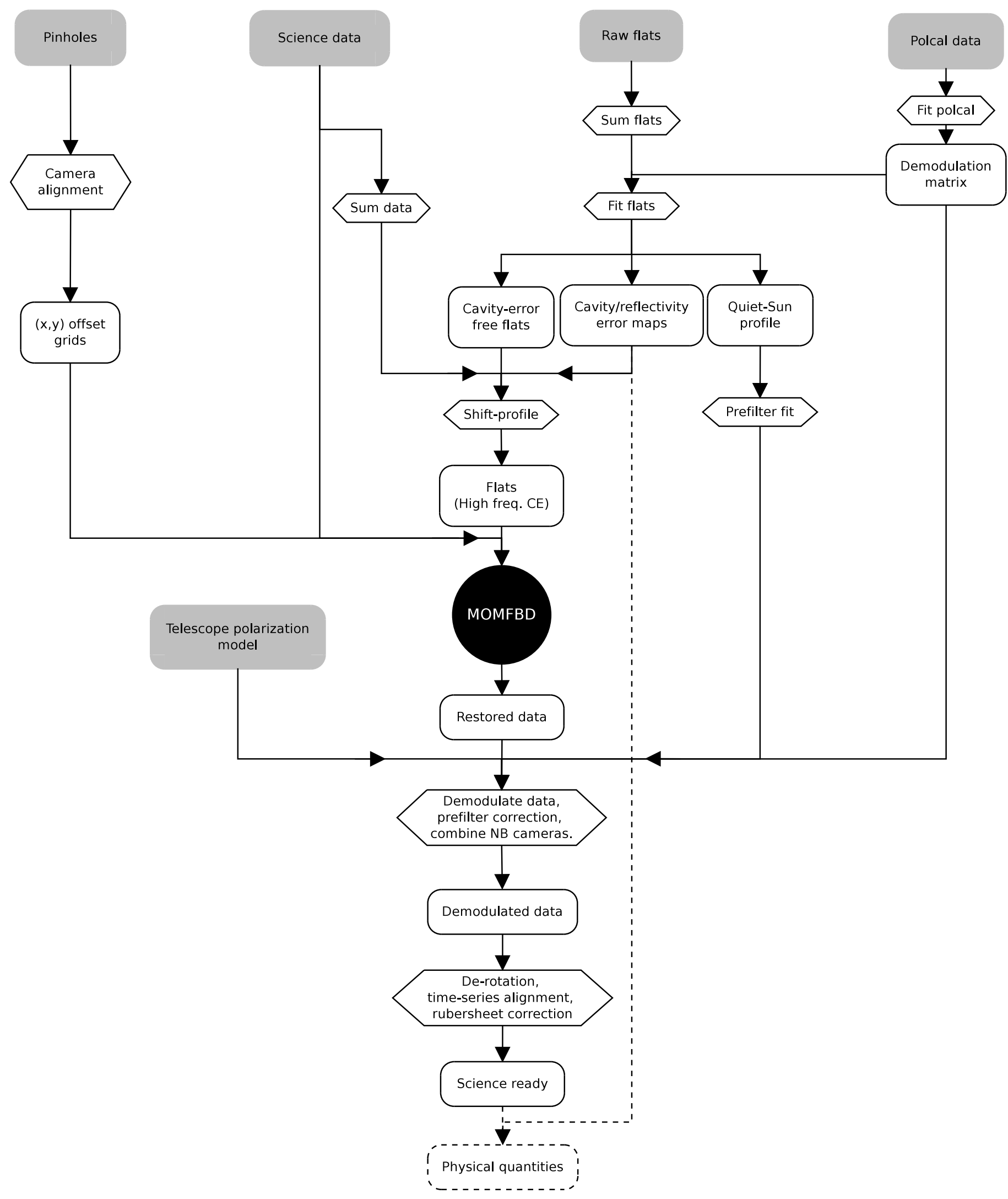

Fig. 9. Data flow in the CRISPRED pipeline. Starting points are the rounded boxes with a gray background: the raw data and the telescope polarization model. The diamond boxes indicate fitting and other processing. Products from fitting routines or data processing are represented by rounded, white boxes. The distinct symbol for the MOMFBD processing indicates that it is not done in IDL. Reflectivity and cavity errors must be included in the interpretation of the science data (outside the CRISPRED pipeline, hence indicated with dashed lines). Omitted in the figure for brevity, all science and calibration images are corrected for dark current. 
The following effects are compensated for within the pipeline. Some effects are coupled as argued in Sect. 3.1, which makes the processing more complicated.

1. Spatial variation in the detector gain and in the zero level offset (bias), IR detector transparency.

2. Spatial variation in the CRISP FPI passband profiles; reflectivity, cavity errors.

3. Optical aberrations from atmospheric turbulence (seeing) as well as from the optics.

4. Inter-camera misalignments.

5. Field-dependent and time-varying instrumental polarization.

6. Image field rotation, temporal misalignment and rubbersheet warping from seeing.

The first steps in a typical reduction are to perform the polarimetric calibration (Sect. 4.1) to derive a demodulation matrix for each pixel. The latter are used to demodulate the flat-field data. We prepare our flat-field images (Sect. 4.2) using spectra from real observations and our processed flats (Sect. 3.1). We use the Stokes I flat-field images to characterize CRISP (Sect. 4.3).

Our two NB cameras are aligned to the WB camera by use of pinhole images (Sect. 3.2), that allow the estimation of offsets in $(x, y)$ positions. These offsets are used by the MOMFBD program in order to make restored images that are well aligned (Sect. 4.4.1).

The restored images are demodulated and compensated for telescope-induced polarization (see Sect. 4.1). Later, we estimate and apply corrections to obtain a homogeneous time-series (Sect. 4.6).

\subsection{Polarimetric calibration}

Above the position of the PCO, the SST has three lenses and four mirrors that modify the polarization properties of the light as a function of pointing angle (and introduce image rotation, see Sect. 4.6). Additionally, the optical table where the CRISP instrument is mounted contains a train of optical elements that also affect polarization (see Fig. 2). We use two modulators to produce four polarization states that are linear combinations of the wanted Stokes parameters. A linearly polarizing beam splitter is used to split the beam in two orthogonal states that are detected simultaneously by each of the NB cameras. This dualbeam setup is necessary for removing, to first order, seeinginduced polarization cross-talk (see Casini et al. 2012, and references therein). For each camera and wavelength, and in each $(x, y)$ pixel, the intensity for the four polarization states can be written as

$\boldsymbol{I}_{\mathrm{obs}}(x, y)=\mathbf{M}_{\mathrm{table}}(x, y) \mathbf{M}_{\mathrm{tel}}(\theta, \varphi) \boldsymbol{I}_{\text {real }}(x, y)$,

where $\boldsymbol{I}_{\mathrm{obs}}$ is the detected intensity vector, $\mathbf{M}_{\mathrm{tel}}$ is the telescope matrix (which depends on the azimuth $\varphi$ and the elevation $\theta$ of the telescope pointing) and $\mathbf{M}_{\text {table }}$ is the modulation matrix and $\boldsymbol{I}_{\text {real }}$ is the real Stokes vector $(I, Q, U, V)$. Selbing (2005) proposed a telescope polarization model for the SST and provided a telescope matrix $\mathbf{M}_{\text {tel }}$ for $630 \mathrm{~nm}$. We use a similar model and up-to-date parameters have been acquired for the prefilters relevant for polarimetry as indicated in Table A.1.

The SST polarization model is constructed using the Mueller matrices of each polarizing element,

$$
\begin{aligned}
\mathbf{M}_{\mathrm{tel}}(\theta, \varphi)= & \mathbf{R}_{f+}\left(a_{11}\right) \cdot \mathbf{N}_{s}\left(a_{9}, a_{10}\right) \cdot \mathbf{N}_{f}\left(a_{7}, a_{8}\right) \\
& \cdot \mathbf{R}_{f-}\left(a_{11}\right) \cdot \mathbf{R}_{\mathrm{az}}(\varphi) \cdot \mathbf{N}_{\mathrm{az}}\left(a_{5}, a_{6}\right) \cdot \mathbf{R}_{\mathrm{el}}(\theta) \\
& \cdot \mathbf{N}_{\mathrm{el}}\left(a_{5}, a_{6}\right) \cdot \mathbf{L}\left(a_{0}, a_{1}, a_{2}, a_{3}, a_{4}\right) .
\end{aligned}
$$

In this model, the entrance lens $(\mathbf{L})$ is a composite of retarders that modulate the light ( 5 free parameters). Mirrors are denoted with $\mathbf{N}$ and have two free parameters. Rotations to a new coordinate frame are indicated with $\mathbf{R}$. The exact form of these matrices is given by Selbing (2005). The properties of the optical elements are wavelength dependent and are contained in the model parameters $a_{0}, a_{1}, \ldots, a_{11}$.

The polarimetric calibration of the optical table is performed as described by van Noort \& Rouppe van der Voort (2008). To acquire the calibration data, known polarization states are generated using a linear polarizer and a quarter-wave plate (QWP hereafter), located just after the exit window of the telescope (PCO in Fig. 2). Typically, for each linear-polarizer angle, the QWP rotates $360^{\circ}$ in $10^{\circ}$ steps. Two linear-polarizer angles suffice to accurately infer the modulation matrix $\mathbf{M}_{\text {table. The pres- }}$ ence of polarizing optics close to some of the focal planes introduces significant field-dependent fluctuations in the elements of the matrix. To account for them, the elements of $\mathbf{M}_{\text {table }}$ are estimated (fitted) for each pixel on the CCD using a multi-threaded C module.

After the MOMFBD process, blurred versions of the demodulation matrix (the inverse of the modulation matrix $\mathbf{M}_{\text {table }}$ ) are applied to the restored images on a pixel by pixel basis as described by Schnerr et al. (2011). Basically, for each NB camera, the demodulation matrix is clipped in the same subfields as the images, then convolved with the average seeing PSF of the corresponding patch and, finally, stitched together in the same way as the restored images. Before applying the demodulation matrix to the data, residual seeing distortions are removed from each of the polarization states as described by Henriques (2012).

Data from the two NB cameras are combined after demodulation and normalization with respect to the mean intensity (Stokes $I$ ), so each contribute with the same weight to the average. This step is necessary because the cameras do not have strictly the same gain.

Telescope polarization is compensated for in a subsequent step, if the parameters of the telescope model are known for the observed spectral region.

\subsection{Detector effects}

The pixel by pixel gain and bias are in principle easy to calibrate. Exposures collected while the beam is blocked (dark frames) establish the bias and flat fields establish the gain. The off-disk sky being too dark for solar setups, flats in solar telescopes are collected as averages of quiet-sun granulation. For the SST, this is done while moving the telescope pointing in a circle over quiet Sun near disk center and blurring the image by putting out random voltages on the DM. Flats are collected individually for each wavelength tuning and polarization state.

Both darks and flats are made by averaging many exposures in order to reduce shot noise and - in the case of flats - reduce the influence of solar structures. We check statistics for all dark and flat frames and remove outliers before summing. This way we essentially remove the risk of having the final flats and darks being destroyed by the occasional camera or shutter glitch, birds flying in front of the telescope aperture, etc.

Bad pixels are zeroed in the gain tables to be read by the MOMFBD program, which interprets the zero gain pixels as instructions to calculate interpolated values for the corresponding pixels in the image data. Isolated or small groups of bad pixels are found with heuristics based on identifying outliers in an unsharp-masked version of the summed flat fields. 
Large CCDs are often divided into regions that are read out separately. We have found that the borders of such regions can cause artifacts in the image restoration, probably due to slight non-linearities that differ between the regions. This is the case for the Sarnoff cameras in the CRISP setup. We routinely mark a column of pixels as bad at such borders, which appears to be an easy remedy for such problems. Because of this, the absolute majority of pixels to be interpolated by the MOMFBD program are oriented in columns. We have therefore implemented an option to the MOMFBD program, to use one-dimensional interpolation across those columns instead of the default circularly symmetrical interpolation. We have found that this further reduces the artifacts from such borders.

A WB observed image is corrected by pixel-by-pixel subtraction of the bias and division with the gain. However, the flats correct not only for detector characteristics, but also for imperfections in the near-focus optical elements, e.g., dust. These effects couple with polarization and are therefore time-dependent for the NB images. The procedure for dealing with this is described in Sect. 3.1 above.

At near-IR wavelengths, the silicon in the detector substrate can become semi-transparent, so that back-scatter from electronics and other camera parts behind the detector adds a component to images that cannot be removed by normal flat-fielding. We have adopted the procedure for removing such effects described by de la Cruz Rodriguez (2010) and de la Cruz Rodríguez et al. (2013, Appendix A.1). The back scatter is modeled with a PSF describing the scattering in the material behind the CCD and a gain factor representing the amount of light that returns from that direction. For our Sarnoff cameras, this procedure is needed at wavelengths longer than $\sim 700 \mathrm{~nm}$ (currently affecting data acquired through the $777 \mathrm{~nm}$ and $854 \mathrm{~nm}$ CRISP prefilters).

\subsection{FPI characterization}

In this section we summarize the methods used to characterize the cavity-error and reflectivity-error maps, and how to estimate and remove the quiet-Sun profile from the flats.

The way flat fields (flats) are made, by averaging many shortexposure images collected while the telescope moves in small circles around the center of the solar disk, allows the assumption that the spectrum present at each pixel corresponds to a spatially averaged quiet-Sun profile at disk center. Therefore, the flats are also affected by the presence of cavity errors, although with a different line profile than line profiles in the observations.

We use a self-consistent method developed by Schnerr et al. (2011). The basic idea is to remove the imprint of spectral lines from the summed flats. The presence of cavity and reflectivity errors, fringes and variations of the prefilter across the FOV, can be understood as a distortion of the profiles, and it can be modeled using a self-consistent iterative scheme, here implemented in an external multi-threaded $\mathrm{C}++$ module that makes use of a Levenberg-Marquardt algorithm to fit the data (cMPFIT, Markwardt 2009).

In the first iteration, cavity errors are unknown, so we estimate the quiet-Sun profile by fitting a cubic non-overshooting Bezier-spline (see summary by Auer 2003) to the average spectrum imprinted in the flats. That average spectrum is shifted in wavelength and scaled in intensity to match the observed spectrum at each pixel, providing a first estimate of the cavity-error map and the gain at each pixel. For stability purposes, in this first iteration, the prefilter variations and the reflectivity are assumed to be zero.
Using the fitted values, we place all spectra (across the FOV) in a large array of $(\lambda, I)$ values, corrected for the cavity-error shift and gain value. A new estimate of the quiet-Sun average is derived by fitting a Bezier-spline to all data points. In the following iterations, a slightly more complete model is used to fit the data, including the gain value, a cavity error, reflectivity fluctuations and prefilter/fringe variations. The latter are fitted using a polynomial multiplicative term to the average spectrum. The model is summarized as

$I(\lambda)=G \cdot \mathfrak{F}^{-1}\left\{\mathfrak{F}\{\hat{I}(\lambda)\} \frac{\mathfrak{F}\{T(\delta \lambda, \delta R)\}}{\mathfrak{F}\{T(0,0)\}}\right\}\left(1+\sum_{n=1}^{N} c_{n} \lambda^{n}\right)$,

where $G$ is the value of the gain, $\hat{I}$ is the estimate of the quietSun average, $T(\delta \lambda, \delta R)$ is the combined FPI transmission profiles of the two etalons (see Eq. (1)), $\delta \lambda$ and $\delta R$ are the cavity and reflectivity errors respectively, and $c_{n}$ are the coefficients of the multiplicative polynomial component. All the fitted parameters are allowed to vary between pixels. We note that $T(0,0)$ is a transmission profile computed using the reflectivity value provided by the FPI manufacturer and no shift. We also note that the length of the polynomial component can be adapted to each case, depending on the wavelength coverage of the observations and how strong fringes are at that wavelength. The $\mathfrak{F}$ operator represents the one-dimensional Fourier transform and $\mathfrak{F}^{-1}$ its inverse. At each pixel, we fit the value of $\delta \lambda, \delta R$ and $c_{1}, \ldots, c_{n}$. Further details are given by Schnerr et al. (2011).

Figure 4 illustrates the resulting reflectivity (top) and cavity (middle) error maps that are inferred using our routines. Additionally, in Fig. 10 we represent a two-dimensional histogram computed from all spectra in the FOV, corrected for cavity errors, normalized by the gain value and corrected for the polynomial component. In this figure we have not corrected the data for reflectivity errors, although those are included in our model. Once all the corrections are applied, we expect all data points to tightly describe the same spectral profile.

Chromospheric lines, like H I $\lambda 6563$ and Ca II 18542 , are much wider than photospheric lines. Under those circumstances, if the slope of the line has a linear behavior within the width of the transmission profile, the imprint of reflectivity variations is extremely mild and this approach fails most of the times, especially when the observations are not critically sampled in the spectral dimension. Instead, the inferred reflectivities are usually dominated by a fringe pattern. Therefore, a simplification to this scheme was proposed by de la Cruz Rodríguez et al. (2013), where reflectivities are assumed to be constant across the FOV and the model can be simplified enormously as convolutions become unnecessary and the mean spectrum can be shifted using cubic Bezier interpolation. This is now the default in CRISPRED, with the reflectivity fitting recommended only when the reflectivities are actually going to be used for the analysis.

\subsection{Image restoration}

\subsubsection{MOMFBD}

Images are restored from wavefront aberrations by means of the Multi-Object Multi-Frame Blind Deconvolution (MOMFBD; van Noort et al. 2005) method, based on the algorithm by Löfdahl (2002). Not using any assumptions about the aberration statistics, the method can compensate for optical aberrations, both from the atmosphere and from the optics, and partial compensation by the AO does not introduce any extra complexity. 


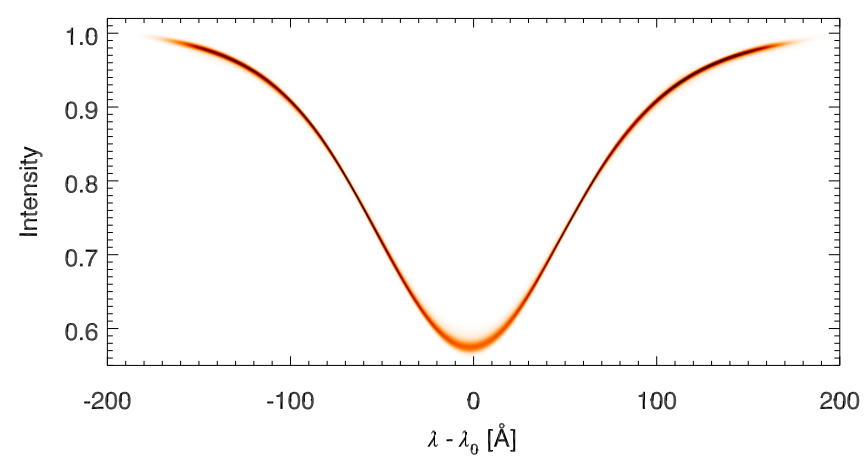

Fig. 10. Two-dimensional histogram of the quiet-Sun spectra at the $\mathrm{Fe}$ I $617.3 \mathrm{~nm}$ line, from the flat field data, where each spectrum (from a different pixel) has been shifted to correct the cavity error and scaled according to the gain value.

However, we do take the aberration statistics into account by using atmospheric Karhunen-Loève modes (KL, Roddier 1990) in the expansion of the pupil phase. These modes are constructed so they are both orthogonal on a circular aperture and statistically uncorrelated assuming atmospheric turbulence following a Kolmogorov model.

The correction is limited by the finite parametrization of the wavefront, so high-order aberrations from both sources are not corrected. The uncorrected aberrations show up in the restored data as a stray light component. By default, we are currently using the 35 most significant atmospheric KL modes as basis functions for expanding the unknown wavefronts. This number was set to about the same as the number of modes corrected by the $\mathrm{AO}$. However, as the $\mathrm{AO}$ was recently upgraded to correcting 85 modes, the default number of KL modes routinely used in MOMFBD will probably be increased.

The MOMFBD algorithm is based on the assumption that the aberrations are isoplanatic, so that the point spread function (PSF) is constant within the FOV. In order to make this assumption hold, at least approximately, the processing is done on overlapping subfields of $\sim 5$ arcsec squared. This makes it possible to find the PSF and deconvolve the raw data by use of fast Fourier transforms. In the process, each subfield is low-pass filtered individually based on the local signal-to-noise ratio. The restored subfields are then mosaicked, resulting in a restored version of the entire FOV.

\subsubsection{Alignment}

Physical quantities like magnetic field strengths and line-of-sight velocities are encoded as small variations in the intensity. Stokes maps and Dopplergrams are calculated as linear combinations of intensity images from different modulator states or wavelength tunings (here: just states). Errors in alignment of the images involved will show up as artifacts in the calculated quantities. However, because the intensity structures differ from state to state, cross correlation methods are not useful for alignment of images from different states.

Given accurate information about the alignment of cameras from the pinhole array (see Sect. 3.2), the MOMFBD joint processing of images from the WB camera with the variable states images from two NB cameras will make all the restored images from a single scan aligned to subpixel precision (van Noort et al. 2005). The reason this works is that all the WB images go into making a single restored image (the anchor image), forcing the estimated wavefronts (mainly the tip and tilt components) to be such that they align the raw WB images. Every NB image is simultaneous to one of the WB images, and their estimated wavefronts are constrained to differ by exactly the fixed tip and tilt offset that align the cameras.

In spite of the subfielding, there are residual anisoplanatic effects in the restored data. Because the raw images for the different NB states are not collected simultaneously, these residuals vary from state to state. This results in relative geometric distortions on scales smaller than the subfields. In the CRISPRED pipeline, we have implemented the improvement suggested by Henriques (2012), capable of removing also such differential stretching from the restored NB images: In addition to the restored WB image based on the entire sequence of raw WB images, the MOMFBD program makes a set of additional restored WB images, each based only on the raw images corresponding to the raw NB images of each state. We then calculate alignment parameters (stretch vectors) on a grid, that align this WB image with the anchor WB image, and apply them to the corresponding restored NB image. We note that this does not remove the variable blurring at scales smaller than the subfields.

\subsection{Prefilter correction}

Most FPI instruments use a prefilter to remove parasitic transmission peaks that are present in the transmission profile. The observed spectra are weighted by the profile of this prefilter, which must be compensated for. In Sect. 4.3 we estimate the spatially averaged quiet-Sun profile from the flat-field data. Similarly to Schnerr et al. (2011), we degrade the FTS solar atlas (Brault \& Neckel 1987) with a theoretical CRISP profile and a theoretical prefilter curve to reproduce our observed quiet-Sun profile.

We adopt a Lorentzian profile to describe the prefilter, with the following parameters

$$
P\left(\lambda ; p_{1}, p_{2}, p_{3}, p_{4}, p_{5}\right)=\frac{p_{1}}{1+\left(2 \cdot \frac{\lambda-p_{2}}{p_{3}}\right)^{2 p_{4}}} \cdot\left(1+\lambda \cdot p_{5}\right),
$$

where $\lambda$ is the wavelength offset from line center, $p_{1}$ is a scaling factor, $p_{2}$ is the central wavelength of the prefilter relative to line center, $p_{3}$ is the FWHM of the prefilter, $p_{4}$ is the number of cavities of the prefilter (normally fixed to 2 ) and $p_{5}$ is a term to account for asymmetries in the prefilter curve. Our implementation allows us to fix the value of some parameters, which can be useful if the number of observed line positions is small or restricted to a small wavelength range.

The estimated prefilter curve is used to correct the intensities of the science data (see Sect. 4.6).

\subsection{Post-processing of time-series}

Image restoration is performed on each line scan individually. Forcing the average of the tip-tilt corrections to be zero yields restored images where the geometric distortions from anisoplanatism are largely removed. However, some jitter may still be present, which becomes apparent when combining many scans into time-series. For the same reason, there can be residual alignment errors between scans. Finally, the alt/az turret introduces image rotation along the day as the relative angle of the mirrors changes within the telescope. In this section we describe how we create homogeneous time-series.

Once the data are restored with MOMFBD and the polarimetric calibration has been applied, we compute time dependent corrections to obtain a homogeneous time series using the WB images. In a second step, we apply those corrections to the NB images to create science-ready data cubes. Polarimetric 
datasets result in sequential cubes with shape $n_{x} \times n_{y} \times n_{\lambda} \times$ $n_{\text {Stokes }} \times n_{t}$, whereas non-polarimetric data have one dimension less, $n_{x} \times n_{y} \times n_{\lambda} \times n_{t}$. The output is compatible with CRISPEX ${ }^{4}$, a versatile tool for the exploration of multi-dimensional datasets by Vissers \& Rouppe van der Voort (2012).

Image field rotation angles are computed theoretically from the position of the Sun on the sky and a telescope pointing model. After de-rotation, the WB images are co-aligned using cross-correlation methods. The residual rubber-sheet motions that usually appear among the different time steps, are removed by resampling following Shine et al. (1994). In short, the idea is to divide the images in small subfields and compute the shift that aligns each subfield with a reference. These corrections are also applied to the cavity map, resulting in a different wavelength correction for each time-step.

Variations in the intensities are expected as the elevation of the Sun changes on the sky. We compute variations in the mean WB intensity during the sequence and compensate for them. The dataset is also corrected for the prefilter curve that we measured in Sect. 4.5.

\section{Discussion}

In this study, we present a new approach to compensate for the effect of cavity errors prior to image restoration. This method is more accurate and fundamentally more correct than the approximation presented in Schnerr et al. (2011), and we advise to always use it to process data from FPI instruments that are mounted in a telecentric setup.

We also present improvements to the pinhole calibration process needed to make the MOMFBD program output restored images from different states that are well aligned, as well as the results of a new procedure for measuring the image scale of image data from any high resolution solar telescope.

We have developed a processing pipeline for reducing data from the SST/CRISP instrument in the form of an objectoriented framework. This is constructed using existing routines and detailed knowledge of the instrument, and produces scienceready datasets from the raw images. However, routines that are specific to other instruments can be easily incorporated in the future, i.e., the forthcoming CHROMIS instrument, a Fabry-Pérot

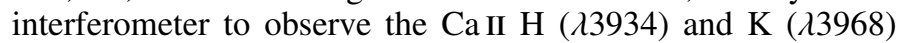
lines. We note that many of the routines included in this framework are also useful as building blocks for building one-shot scripts for unusual datasets.

The transmission profiles of the FPI etalons were computed (see Sect. 2.3) assuming perpendicular incidence of the light. In reality, the beam is slowly converging and the low resolution etalon is tilted to reduce ghost images. Both the tilt angle and the extreme angles from the beam convergence are $\arctan (1 / 165) \approx 0.17$. These two effects in practice make the total CRISP profile slightly wider and sightly asymmetric. For simplicity, we have adopted the simplified description of Eq. (1), but we plan to investigate those effects in the future and include compensation for them in the pipeline if necessary.

Some known effects are currently not included in the pipeline. The finite area of the detector pixels causes a slight reduction of signal at the highest detected spatial frequencies. The corresponding modulation transfer function (MTF) also includes a wavelength dependent component from charge diffusion that is more difficult to model (Stevens \& Lavine 1994). Compensation

\footnotetext{
4 Now included in Solarsoft and available on the web at http:// folk.uio.no/gregal/crispex/
}

for the geometric part of the detector MTF would not be difficult to add but the wavelength-dependent charge-diffusion effect requires either careful calibrations or information from the manufacturer that we do not have. There is ongoing work for the characterization of the various sources of straylight at the SST and removing their effects (Scharmer et al. 2010; Löfdahl $\&$ Scharmer 2012). When we have a good enough model for this, a correction may be added to the pipeline.

The way flat fields (flats) are made, by averaging many shortexposure images collected while the telescope moves in small circles around the center of the solar disk, allows the assumption that the spectrum present at each pixel corresponds to a spatially averaged quiet-Sun profile at disk center. The presence of active regions can sometimes be inconvenient, forcing the observer to trace more elliptical trajectories. To avoid distortions in the quiet-Sun profile, one could normalize the NB flats by use of the mean intensity of the WB flat images (acquired simultaneously with the NB images) as they should average to the same value for all wavelengths. We are planning to implement this as an optional step.

As part of the SOLARNET project, there is an effort to prepare for the inclusion of data from ground-based telescopes in a virtual solar observatory, similar to what is done for data from space-based solar telescopes. Recommendations for file formats and meta data are expected in the fall of 2014 and we aim to make CRISPRED compliant as soon as possible.

Acknowledgements. We thank G. Scharmer and D. Kiselman for illuminating discussions. The Swedish $1 \mathrm{~m}$ Solar Telescope is operated on the island of La Palma by the Institute for Solar Physics in the Spanish Observatorio del Roque de los Muchachos of the Instituto de Astrofísica de Canarias. This work was carried out as a part of the SOLARNET project, funded by the European Commission's 7th Framework Programme under grant agreement No. 312495. We use subroutines from the IDL astronomy User's Library (Landsman 1993). IDL $^{\circledR}$ is a trademark of Exelis Inc. This research has made use of NASA's Astrophysics Data System (ADS).

\section{Appendix A: CRISP prefilters}

Currently ten prefilters are available to observe selected spectral lines, which have been indicated in Fig. A.1 and detailed in Table A.1. Selection of the appropriate prefilter for each observing sequence is done by means of a filter wheel that is located near a focal plane (see Fig. 2). The central wavelength (CWL) of the filters are slightly to the red of the line core for which

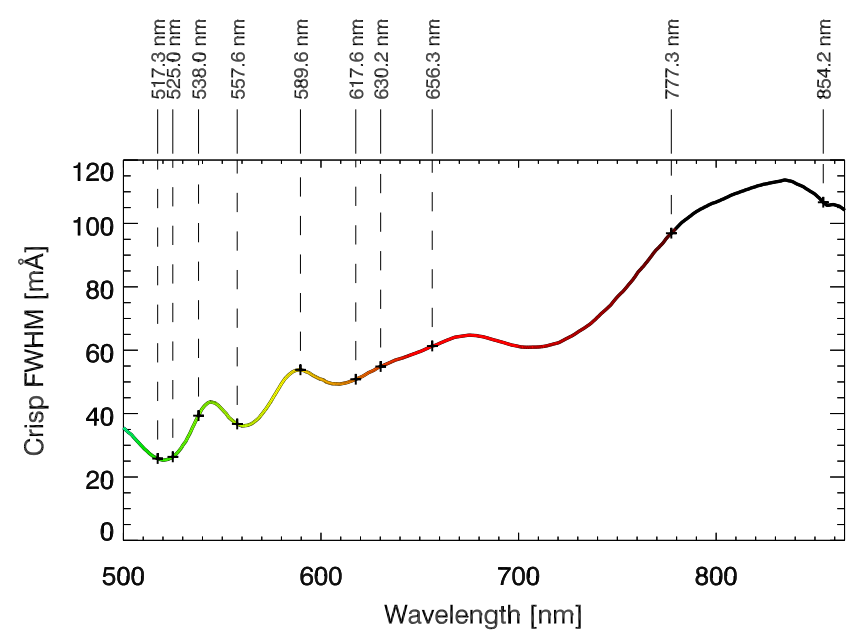

Fig. A.1. FWHM of the CRISP transmission profile as a function of wavelength. We have indicated those lines that are currently observable (i.e., a prefilter is available). 
Table A.1. List of prefilters for the CRISP instrument.

\begin{tabular}{|c|c|c|c|c|}
\hline \multirow{2}{*}{$\frac{\text { CWL [nm] }}{517.33}$} & \multirow{2}{*}{$\begin{array}{c}F W H M[\mathrm{~nm}] \\
0.30\end{array}$} & \multicolumn{2}{|c|}{ Main diagnostic } & \multirow{2}{*}{ PC } \\
\hline & & $\operatorname{Mg}_{I}$ & 517.2 & \\
\hline 525.01 & 0.30 & $\mathrm{Fe} I$ & 525.0 & $\checkmark$ \\
\hline 538.20 & 0.34 & $\mathrm{CI}_{\mathrm{I}}$ & 538.0 & $\checkmark$ \\
\hline 557.80 & 0.30 & $\mathrm{Fe} I$ & 557.6 & \\
\hline$* 587.6$ & 0.4 & $\mathrm{He} \mathrm{I}$ & 587.6 & \\
\hline 589.70 & 0.39 & $\mathrm{NaI}$ & 589.6 & \\
\hline 617.39 & 0.42 & $\mathrm{Fe} I$ & 617.3 & $\checkmark$ \\
\hline 630.26 & 0.44 & $\mathrm{Fe} \mathrm{I}$ & 630.2 & $\checkmark$ \\
\hline 656.38 & 0.49 & H I & 656.3 & \\
\hline 777.24 & 0.77 & OI & 777.2 & $\checkmark$ \\
\hline 854.16 & 0.93 & Ca II & 854.2 & $\checkmark$ \\
\hline
\end{tabular}

Notes. ${ }^{(*)}$ Ordered from the manufacturer at the time of this publication. PC: Calibrated for polarization effects, see Sect. 4.1.

they are designed. The whole filter wheel sits on a motorized tiltable mount, which allows tuning of the filters to the exact wavelengths. In some cases this allows easy observation of other interesting spectral lines close to the primary one.

\section{References}

Auer, L. 2003, in Stellar Atmosphere Modeling, eds. I. Hubeny, D. Mihalas, \& K. Werner, ASP Conf. Ser., 288, 3

Bello González, N., \& Kneer, F. 2008, A\&A, 480, 265

Brault, J. W., \& Neckel, H. 1987, Spectral Atlas of Solar Absolute Diskaveraged and Disk-Center Intensity from 3290 to $12510 \AA$, ftp: //ftp. hs . uni-hamburg.de/pub/outgoing/FTS-Atlas/

Casini, R., de Wijn, A. G., \& Judge, P. G. 2012, ApJ, 757, 45

Cauzzi, G., Reardon, K. P., Uitenbroek, H., et al. 2008, A\&A, 480, 515

Cavallini, F. 2006, Sol. Phys., 236, 415

de la Cruz Rodriguez, J. 2010, Ph.D. Thesis, Stockholm University, http: //urn.kb.se/resolve?urn=urn:nbn: se: su:diva-43646

de la Cruz Rodríguez, J., Rouppe van der Voort, L., Socas-Navarro, H., \& van Noort, M. 2013, A\&A, 556, A115

De Pontieu, B., Carlsson, M., Rouppe van der Voort, L. H. M., et al. 2012, ApJ, $752, \mathrm{~L} 12$

de Wijn, A. G., Tomczyk, S., Casini, R., \& Nelson, P. G. 2011, in Solar Polarization 6, eds. J. R. Kuhn, D. M. Harrington, H. Lin, et al., ASP Conf. Ser., 437, 413

Dunion, J. P., \& Velden, C. S. 2004, Bull. Am. Meteorol. Soc., 85, 353

Fried, D. L. 1965, J. Opt. Soc. Am., 55, 1427

Henriques, V. M. J. 2012, A\&A, 548, A114

Kentischer, T. J., Schmidt, W., Sigwarth, M., \& Uexkuell, M. V. 1998, A\&A, 340,569

Landsman, W. B. 1993, in Astronomical Data Analysis Software and Systems II, eds. R. J. Hanisch, R. J. V. Brissenden, \& J. Barnes, ASP Conf. Ser., 52, 246, see also http: //idlastro.gsfc.nasa.gov
Leenaarts, J., de la Cruz Rodríguez, J., Kochukhov, O., \& Carlsson, M. 2014, ApJ, 784, L17

Löfdahl, M. G. 2002, in Image Reconstruction from Incomplete Data II, eds. P. J. Bones, M. A. Fiddy, \& R. P. Millane, Proc. SPIE, 4792, 146

Löfdahl, M. G., \& Scharmer, G. B. 2012, A\&A, 537, A80

Markwardt, C. B. 2009, in Astronomical Data Analysis Software and Systems XVIII, eds. D. A. Bohlender, D. Durand, \& P. Dowler, ASP Conf. Ser., 411, 251

Martínez Pillet, V., Del Toro Iniesta, J. C., Álvarez-Herrero, A., et al. 2011, Sol. Phys., 268, 57

Norén, A. 2013, Bachelor's thesis, Stockholm University, http://arxiv.org/abs/1311.5148

Puschmann, K. G., Denker, C., Balthasar, H., et al. 2013, Opt. Eng., 52, 1606

Reardon, K. P., \& Cavallini, F. 2008, A\&A, 481, 897

Reardon, K. P., Rimmele, T., Tritschler, A., et al. 2009, in The Second Hinode Science Meeting: Beyond Discovery-Toward Understanding, eds. B. Lites, M. Cheung, T. Magara, J. Mariska, \& K. Reeves, ASP Conf. Ser., 415, 332

Roddier, F. 1999, in Adaptive Optics in Astronomy, ed. F. Roddier (Cambridge: Cambridge university press), 25

Roddier, N. 1990, Opt. Eng., 29, 1174

Rouppe van der Voort, L., \& de la Cruz Rodríguez, J. 2013, ApJ, 776, 56

Scharmer, G. B. 2006, A\&A, 447, 1111

Scharmer, G. B., \& van Werkhoven, T. I. M. 2010, A\&A, 513, A25

Scharmer, G. B., Dettori, P., Löfdahl, M. G., \& Shand, M. 2003, in Innovative Telescopes and Instrumentation for Solar Astrophysics, eds. S. Keil, \& S. Avakyan, Proc. SPIE, 4853, 370

Scharmer, G. B., Narayan, G., Hillberg, T., et al. 2008, ApJ, 689, L69

Scharmer, G. B., Löfdahl, M. G., van Werkhoven, T. I. M., \& de la Cruz Rodríguez, J. 2010, A\&A, 521, A68

Scharmer, G. B., de la Cruz Rodriguez, J., Sütterlin, P., \& Henriques, V. M. J. 2013, A\&A, 553, A63

Schnerr, R. S., de La Cruz Rodríguez, J., \& van Noort, M. 2011, A\&A, 534, A45

Schou, J., Scherrer, P. H., Bush, R. I., et al. 2012, Sol. Phys., 275, 229

Sekse, D. H., Rouppe van der Voort, L., De Pontieu, B., \& Scullion, E. 2013, ApJ, 769, 44

Selbing, J. 2005, Master's thesis, Stockholm University, http: //arxiv.org/abs/1010.4142

Shine, R. A., Title, A. M., Tarbell, T. D., et al. 1994, ApJ, 430, 413

Steiner, O., Franz, M., Bello González, N., et al. 2010, ApJ, 723, L180

Stevens, E. G., \& Lavine, J. P. 1994, IEEE Trans. electronic devices, 41, 1753

Tritschler, A., Schmidt, W., Langhans, K., \& Kentischer, T. 2002, Sol. Phys., 211,17

Tritschler, A., Schlichenmaier, R., Bellot Rubio, L. R., et al. 2004, A\&A, 415, 717

van Noort, M. 2012, A\&A, 548, A5

van Noort, M. J., \& Rouppe van der Voort, L. H. M. 2008, A\&A, 489, 429

van Noort, M., Rouppe van der Voort, L., \& Löfdahl, M. G. 2005, Sol. Phys., 228, 191

Vissers, G., \& Rouppe van der Voort, L. 2012, ApJ, 750, 22

Vissers, G. J. M., Rouppe van der Voort, L. H. M., \& Rutten, R. J. 2013, ApJ, 774,32

Watanabe, H., Bellot Rubio, L. R., de la Cruz Rodríguez, J., \& Rouppe van der Voort, L. 2012, ApJ, 757, 49

Wedemeyer, S., Scullion, E., Rouppe van der Voort, L., Bosnjak, A., \& Antolin, P. 2013, ApJ, 774, 123 